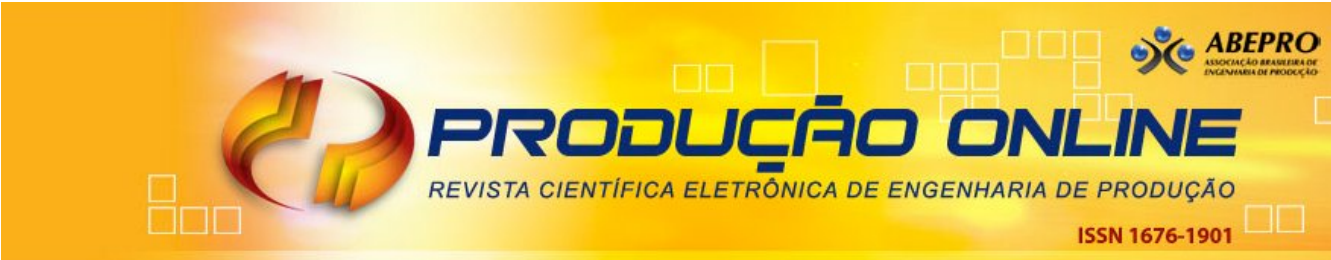

\title{
ESTUDO COMPARATIVO ENTRE LAYOUTS SOB A ÓTICA DA TEORIA DAS RESTRIÇÕES COM APOIO DE SIMULAÇÃO DE EVENTOS DISCRETOS EM EMPRESA DE ALIMENTOS
}

\section{COMPARATIVE STUDY OF LAYOUTS FROM THE PERSPECTIVE OF THE THEORY OF CONSTRAINTS WITH SUPPORT OF DISCRETE EVENT SIMULATION IN A FOOD COMPANY}

\author{
Lucas Eggers Assunpção* E-mail: lucas-eggers@hotmail.com \\ William Jacobs* E-mail: williami@univates.br \\ *Universidade do Vale do Taquari (UNIVATES), Lajeado, RS
}

\begin{abstract}
Resumo: O layout tem interferência direta nos sistemas produtivos das empresas. Contudo não é tarefa fácil prever todas as variáveis em seu rearranjo, e se ao final atenderá às metas globais da empresa. Buscando contribuir com esta questão o presente estudo propôs uma nova abordagem para análise de layout, empregando a simulação de eventos discretos e os indicadores operacionais da Teoria das Restrições (TOC), para avaliar qual o arranjo físico mais adequado - celular ou funcional - a ser utilizado em um setor de misturas de uma empresa de alimentos. Esta análise foi realizada sob a perspectiva dos seguintes indicadores operacionais da TOC: ganho, investimentos e despesas operacionais. Inicialmente foram identificados os produtos mais vendidos em um período de 12 meses, por meio da curva $A B C$. Em seguida foram realizados os projetos de layout: funcional, através do método de planejamento computadorizado de relação de layout (CORELAP); celular, através da análise de fluxo de produção (FPA). Depois de realizada a modelagem dos layouts com os dados coletados, as simulações foram rodadas e posteriormente analisadas com base nos indicadores operacionais da TOC, apontando ao final o layout mais adequado. Os resultados mostraram que o layout funcional apresentou o melhor desempenho sob a perspectiva dos indicadores operacionais da TOC, proporcionando o aumento no ganho $(+12,34 \%)$, redução nos investimentos $(-11,47 \%)$ e redução nas despesas operacionais $(-18,71 \%)$. Este estudo demonstrou que o layout funcional, no contexto estudado, ofereceu maior flexibilidade produtiva, impactando de forma positiva nos indicadores operacionais da TOC e que tais indicadores podem ser ferramentas efetivas na comparação de layouts.
\end{abstract}

Palavras-chave: Layout. Simulação de eventos discretos. Teoria das restrições. Indicadores de desempenho.

Abstract: The layout exerts important interferences in the manufacturing systems of the companies. However, it is not an easy task to predict all the variables in layout rearrangement, and whether it will ultimately meet the company goals. In order to contribute to this issue, the present study proposed a new approach for layout analysis, using the discrete event simulation and the operational indicators of Theory of Constraints (TOC), to evaluate the most appropriate layout - job shop or cellular - to be used in a food company. This analysis was performed with the TOC indicators: gain, investments and operating expenses. First the best-selling products in a period of 12 months were identified with the $A B C$ curve. Then the layout projects were carried out: the job shop layout design was performed using the Computerized Relationship Layout Planning (CORELAP) method, and the cellular layout design through the production flow analysis (FPA). After the modeling of the layouts with the collected data, the simulations were carried out and analyzed based on the TOC operational indicators pointing out the most appropriate layout. The results showed that the job shop layout presented the best performance at the perspective of TOC operational indicators, providing an increase in the gain $(+12,34 \%)$, a reduction in investments $(-11,47 \%)$ and a reduction in operating expenses $(-18,71 \%)$. This study demonstrated that the job shop layout, in the context studied, offered greater productive flexibility, 
positively impacting the operational indicators of TOC and that such indicators can be effective tools in the comparison of layouts.

Keywords: Layout. Discrete Event Simulation. Theory Of Constraints. Performance Indicators.

\section{INTRODUÇÃO}

A competitividade empresarial tem trazido novos desafios à produção, como o aumento da variedade de produtos, menor tolerância quanto aos atrasos e imprecisões, redução dos custos de produção, além de um padrão de qualidade mais alto (BLACK, 1998), o que exige novas ferramentas capazes de atender a estas demandas. Um dos fatores com grande impacto na produção é o layout, ou arranjo físico da área produtiva. O tipo de layout afeta o desempenho dos sistemas de produção, sendo dedicado um grande esforço para desenvolver arranjos que atendam às prioridades competitivas conforme o plano de negócios. O planejamento do arranjo físico pode ser de nível estratégico, quando há proposta de grandes mudanças, novas fábricas e grandes ampliações, e de nível tático, quando as mudanças não são tão representativas (PEINADO; GRAEML, 2007).

Para rearranjar ou projetar o layout as empresas geralmente enfrentam duas dificuldades principais - o alto investimento e a imprevisibilidade, durante o planejamento, de todas as variáveis envolvidas no processo produtivo. Umas das formas de planejar um novo layout, com maior previsibilidade e menores custos, é a simulação computacional, a qual consegue envolver todas as variáveis e restrições, além de poder conduzir experimentos sem a necessidade de interromper os sistemas reais (PEINADO; GRAEML, 2007).

Assim, considerando a importância da escolha de um layout o objetivo deste trabalho foi avaliar o arranjo físico mais adequado - celular ou funcional - com apoio da simulação de eventos discretos, a ser utilizado em uma empresa de alimentos sob a ótica dos indicadores operacionais da Teoria das Restrições (TOC): ganho, investimentos e despesas operacionais.

\section{REFERENCIAL TEÓRICO}

A seguir serão abordados alguns aspectos teóricos sobre os tipos de layout mencionados, sobre os indicadores de desempenho da TOC e sobre a simulação 
computacional no contexto de avaliação de layouts.

\subsection{Layout}

Para Slack et al. (2009), o layout ou arranjo físico consiste na configuração em que estão distribuídos os recursos transformadores - onde estão todas as instalações, equipamentos, pessoal de operação e máquinas. Por conseguinte, também consiste na maneira como os recursos transformados fluem pela operação. Assim, pequenas mudanças no layout, como a localização de uma máquina, podem ter consequências sobre o fluxo.

Considerando a importância do layout para que as indústrias funcionem de maneira eficiente, deve-se projetar adequadamente o arranjo físico e constantemente revisar este projeto, permitindo a otimização do fluxo de pessoas, materiais e equipamentos através do sistema. O planejamento de layout tem como principal objetivo a minimização dos custos de processamento e desperdícios de tempo e materiais, e a máxima utilização do processo produtivo (GAITHER; FRAZIER, 2002). A correta decisão do arranjo físico é importante para evitar resultados insatisfatórios, como perdas na produção, alto lead time e custos elevados (SLACK et al., 2009). Além de abranger prioridades estratégicas para a competitividade, qualidade e capacidade produtiva dos processos (KRAJEWSKI; RITZMAN; MALHOTRA, 2009).

Krajewski, Ritzman e Malhotra (2009) citam três diferentes tipos de layout, sendo eles: de posição fixa ou project shop; por produto, linha ou flow shop; e por processo, funcional ou job shop. Para Black (1998), existe ainda o layout em célula, que é obtido com a composição dos layouts funcional e por produto.

A combinação de volume de produção e variedade de produtos são critérios fundamentais na escolha do tipo de layout. Em casos de grande variedade de produtos e baixo volume, como é o caso da empresa de alimentos estudada neste trabalho, indica-se a aplicação dos layouts celular e funcional (SLACK et al., 2009; ASKIN; GOLDBERG, 2002), os quais receberão detalhamento adiante.

\subsubsection{Layout Funcional}

No sistema de layout funcional os equipamentos são dispostos em por função, 
como por exemplo, agrupam-se todas as fresadoras, todos os tornos etc. Isto faz com que os recursos transformados sejam encaminhados em pequenos lotes para cada agrupamento de máquinas, gerando alto nível de estoque no processo (BLACK, 1998). Os produtos percorrem o trajeto pelos grupos de máquinas que lhes são necessários para a sua transformação. Diferentes produtos percorrem roteiros diferentes porque necessitam de grupos de máquinas distintas, podendo provocar um fluxo de operações complexo de ser gerenciado (SLACK et al., 2009).

O arranjo físico funcional é flexível em termos de variedade de produtos devido à localização das máquinas não depender de requisitos dos produtos. Máquinas e outros recursos são agrupados de acordo com a natureza do processo, e os produtos fluem livremente de recurso a recurso, conforme necessário. Outras principais vantagens deste arranjo físico são o elevado grau de especialização dos trabalhadores e supervisores do processo produtivo, maior facilidade de manter a produção ininterrupta por fatores de ausência de funcionário ou quebra de máquina e a facilidade na supervisão de áreas (SLACK et al., 2009). Normalmente o arranjo físico funcional é adotado para atendimento das necessidades do mercado quando há produtos diversificados. No entanto, as principais características deste layout - isto é, baixa capacidade produtiva e alta variedade de produtos - podem trazer dificuldades no fluxo de materiais em processo (LI 2005; DINIS-CARVALHO; ALVEZ; SOUSA, 2014). Este arranjo físico também tem maior incidência de setups, maior complexidade no fluxo de materiais em relação aos demais layouts, o que dificulta o seu controle e traz a necessidade de maior espaço físico e capital para a alta quantidade de estoque em processo (NEUMANN; SCALICE, 2015).

Ainda que a experiência, a intuição e a criatividade sejam fatores importantes no planejamento de arranjo físico, é imprescindível para o projeto de layout aplicação de meios racionais, facilitado pelas abordagens sistemáticas (SANTOS; GOHR; URIO, 2014; SILVA et al., 2015). Uma das técnicas utilizadas para projetar o layout funcional é o CORELAP (Computerized Relationship Layout Planning -Planejamento computadorizado de relação de layout), através da qual classifica-se a proximidade entre os departamentos, quanto ao grau de importância, visando determinar o quão necessário é a proximidade entre eles (GAITHER; FRAZIER, 2002; MOREIRA, 2008). 


\subsubsection{Layout celular}

O layout celular consiste no agrupamento de máquinas que atendem parcial ou completamente às demandas de um determinado grupo de produtos, os quais necessitam dos mesmos recursos. Os produtos são aglutinados conforme necessidade de máquinas para sua produção, sendo chamados de famílias de produtos. Já as máquinas são agrupadas e preparadas para atender aquela família (BURBIDGE, 1981; WEMMERLÖV; JOHNSON, 1997; SLACK et al., 2009).

O arranjo físico celular tem maior flexibilidade quanto ao tamanho dos lotes de produção, já que quando as células atendem a determinada família de produtos os tempos de setup unitários tendem a diminuir, possibilitando assim a redução do tamanho dos lotes de produção (PEINADO; GRAEML, 2007). Há uma melhor relação humana neste arranjo físico, de modo que os trabalhadores formam pequenas equipes de trabalho para a produção completa do produto. Assim, obtém-se maior habilidade dos operadores, de maneira que os funcionários visualizam a produção do início ao fim, e também há uma menor quantidade de estoques em processo (CHASE; JACOBS; AQUILANO, 2006). Outros fatores positivos são a facilidade na mudança de equipamentos da célula, redução nos períodos de treinamento dos trabalhadores, no custo de manuseio de materiais, o no tempo de produção, e maior facilidade de automatização (GAITHER; FRAZIER, 2002).

Para o planejamento do layout celular é amplamente utilizada a Tecnologia de Grupo (TG). A TG agrupa os produtos com características similares em famílias e indica grupos de máquinas para a produção desses itens. As famílias podem ser definidas por forma, tamanho ou demanda. O objetivo, dentre outros, consiste em identificar um grupo de produtos que minimize a necessidade de preparações de máquinas (CORRÊA; CORRÊA, 2006; RITZMAN; KRAJEWSKI, 2004; GAITHER; FRAZIER, 2002; BLACK, 1998; GROOVER, 2001). Burbidge (1975) propôs uma das abordagens mais antigas para a realização da TG, a análise de fluxo de produção ou, em inglês, production flow analysis (FPA). Esta técnica permite identificar os equipamentos necessários para processamento de cada produto, para que em seguida se construa uma matriz relacionando os produtos com seus respectivos equipamentos necessários. Por fim, deve-se analisar e separar em grupos os produtos que utilizam os equipamentos em comum (BLACK, 1998). 


\subsection{Indicadores de desempenho da Teoria das Restrições (TOC)}

A implantação de sistemas de produção exige a correlação com indicadores de custos e desempenho a fim de evitar a perda de credibilidade no resultado efetivo (DIAS et al., 2007). Goldratt (1996) desenvolveu uma forma sistemática para identificação, análise e solução de problemas baseado em um método científico, com relação do tipo efeito-causa-efeito. Segundo Goldratt \& Cox (2002), "a soma dos ótimos locais não é igual à soma dos ótimos globais". Esta afirmação remete a questionamentos como: qual é o ótimo global de uma empresa? Como saber se a meta está sendo alcançada? Como definir as ações locais visando o alcance da meta? A partir desta sistematização foi desenvolvido um sistema de indicadores globais com a função de orientar as ações e resultados das melhorias implantadas. São estes indicadores: lucro líquido (LL), retorno sobre o investimento (RSI) e caixa (C). Estes indicadores globais são avaliados por agentes em níveis hierárquicos elevados da empresa, como por exemplo, diretores. Com a finalidade de trazer estes indicadores para a aplicação em nível gerencial fabril, propuseram-se três indicadores operacionais: o ganho (G), os investimentos (I) e as despesas operacionais (DO) (GOLDRATT; FOX, 1989; GOLDRATT; COX, 2002).

O ganho é o indicador que representa a taxa de geração de dinheiro através das vendas. O investimento (I) ou inventário é todo o dinheiro investido para transformar o produto final em ganho (TOLLINGOTN, 1998; GOLDRATT; COX, 2002; CHASE; JACOBS; AQUILANO, 2006) e a despesa operacional está relacionada com todo o custo e despesas para transformar matéria-prima em produto acabado (ex. salários, aluguel, energia) (GOLDRATT; COX, 2002; CHASE et al., 2006). O indicador ganho tem maior importância na escala de prioridades na Teoria das Restrições, de modo que as empresas devem priorizar o aumento dos ganhos em detrimento à redução dos custos, os quais devem ficar em segundo plano (GOLDRATT, 1996). Goldratt também afirma que a redução dos investimentos é mais importante do que a redução das despesas operacionais, com base no que os japoneses demonstraram sobre a importância da redução dos inventários de produtos acabados e em processo, dado que os investimentos podem incrementar os ganhos futuros na empresa (ANTUNES et al., 2008). 
Uma das questões relevantes sobre alteração de layout é como mensurar se as alternativas propostas estão alinhadas a sua meta global. Para isto foi escolhido utilizar estes indicadores de desempenho, pois permitem avaliar se as ações locais empreendidas estão alinhadas aos objetivos maiores da organização.

\subsection{Simulação computacional}

A simulação computacional consiste na utilização de determinadas técnicas matemáticas com auxílio de computadores digitais, que possibilita imitar o funcionamento de qualquer processo ou operação de um sistema do mundo real (BANKS, 1998; FILHO, 2001; BATEMAN et al., 2013; CHWIF; MEDINA, 2010). Os modelos de simulação possibilitam avaliar o processo de produção e sua evolução ao longo do tempo, sendo uma técnica muito útil quando há limitações de recursos e tempo (DE LIMA, et al., 2016; PEREIRA; COSTA, 2012).

Para Chwif e Medina (2010) o modelo de simulação é comumente utilizado como uma abordagem para obter respostas quando há mudança das variáveis, como por exemplo, frente à adição de máquinas ao processo, redução do número de colaboradores da equipe, ou aumento na demanda. As respostas podem ser inúmeras, assim um modelo de simulação pode ser útil na análise das diversas variáveis que envolvem o sistema.

A simulação computacional tem como uma de suas vantagens o estudo de sistemas complexos com acontecimentos probabilísticos, os quais não podem ser tratados adequadamente por meio de técnicas analíticas, mas podem ser, na maioria das vezes, estudados via simulação. Fornece um controle maior sobre as condições experimentais do que seria possível na experimentação do sistema real e permite a replicação precisa dos experimentos, podendo-se testar cenários para o sistema. Permite também simular longos períodos em um tempo reduzido. Em geral, é mais econômico do que testar o sistema real. Como desvantagens tem-se que a simulação computacional depende da validade do modelo desenvolvido, sendo necessário que o cenário represente fidedignamente o sistema. A técnica da simulação não é por si só otimizante, testando somente as alternativas dadas pelo usuário (LAW; KELTON, 1991). Apesar disto vários autores já mostraram a aplicabilidade da simulação computacional na reformulação de projetos de layout. Meirelles et al. (2009) utilizaram 
a simulação computacional para o estudo de propostas de alterações de layout em uma fábrica de refratários, de forma que a simulação permitiu a fácil realização de alterações; Boblitz (1991) estudou aquisições de novas máquinas e a substituição de suas células de manufatura por uma linha de produção contínua gerando a economia de U\$ 80.000 ao verificar que tal aquisição não traria resultados positivos; Almeida et al. (2006), por meio de simulação realizaram o balanceamento da célula de montagem em uma fábrica de calçados possibilitando a visualização do funcionamento de uma linha produtiva a um baixo custo e auxiliando na tomada de decisão; ainda, Lopes (1999) utilizou a simulação para avaliar a capacidade de fluxo em um sistema de armazenagem de uma fábrica de pneus.

A seguir são apresentados os aspectos metodológicos do trabalho.

\section{METODOLOGIA}

Pesquisa de abordagem quantitativa, cuja coleta e registros dos dados foram realizados in loco - uma empresa do ramo alimentício e limitado apenas a uma das linhas, a saber, a linha de misturas, a qual tem uma grande variedade de produtos. As etapas desenvolvidas neste trabalho (Figura 1) foram baseadas na metodologia proposta por Xavier et al. (2010), com adaptações nas etapas de concepção e análise, predominantemente no mapeamento do processo, escolha do mix de produtos, planejamento de layout e análise através dos indicadores da TOC. 
Figura 1 - Etapas percorridas para realização do trabalho

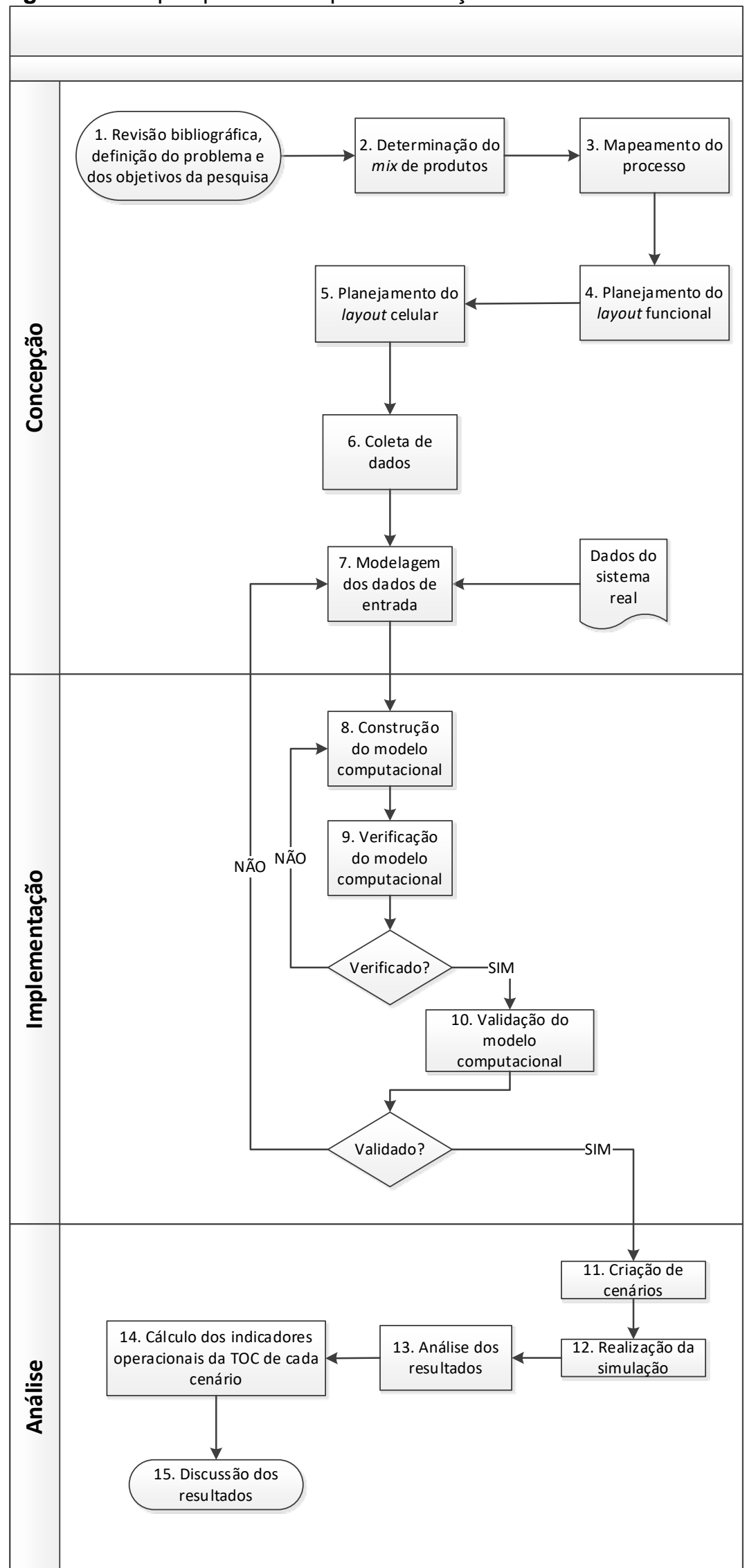

Fonte: Adaptado de Xavier et al. (2010, p. 4)

Revista Produção Online. Florianópolis, SC, v. 19, n. 1, p. 152-178, 2019. 
A partir de revisão bibliográfica, definiu-se o problema e os objetivos do estudo (1). A seguir foram definidos os produtos de maior representatividade por volume (mix produtivo) com o auxílio da curva $A B C$ (2) e mapearam-se as práticas de gerenciamento e o fluxo produtivo, a partir de observações, entrevistas e análise documental (3). Projetou-se então o layout funcional, por meio do método de planejamento computadorizado de relação de layout (CORELAP) (4), e o projeto de layout celular, através do método de análise de fluxo de produção (FPA) (5). Coletouse o tempo de cada operação dos produtos bem como foram listados os recursos, equipamentos e suas respectivas capacidades (6). Os tempos foram analisados através do software Statfit ${ }^{\circledR}$ para verificar qual a distribuição contínua probabilística mais adequada a ser aplicada em cada grupo de amostras coletadas e seu grau de dispersão (7). A partir destes dados e do número de operadores das linhas, de equipamentos, tempos de espera, taxa de refugos e programação dos turnos (horário de início, término e paradas programadas) construiu-se um modelo computacional do cenário atual. Para isso utilizou-se o software ProModel ${ }^{\circledR}$ (8). As verificações deste modelo foram realizadas ao longo do processo e corrigidas quando necessário (9); os dados foram validados através do comparativo entre a quantidade produzida do sistema real e do modelo computacional do cenário atual, buscando menor índice percentual de diferença (10). A partir daí foi possível construir os modelos do layout funcional e celular e mensurar os dados para o cálculo dos indicadores operacionais da TOC (11), além de realizar as simulações do comportamento de cada layout, sendo cada um deles replicado cinco vezes (ARAÚJO, 2002; PINHO et al., 2006; SOARES et al., 2011). Cada uma das replicações teve o tempo de 8 horas e 48 minutos, um dia de trabalho do setor produtivo (12). Os resultados obtidos foram analisados e os cenários alterados buscando-se a otimização dos modelos computacionais (13). A partir destes e dados da contabilidade foram calculados os valores dos indicadores de desempenho da TOC (14) e realizada a discussão geral dos resultados (15).

\subsection{Estudo de caso}

Este capítulo faz uma descrição da empresa onde é realizado o estudo, da linha de manufatura e dos produtos fabricados. Também apresenta uma aplicação prática 
do método proposto para realizar a análise de layouts, e sua discussão, atendendo aos objetivos do estudo.

\subsection{O ambiente produtivo}

Como campo de estudo, escolheu-se uma linha de produção dedicada à fabricação exclusiva de misturas alimentícias. Este setor é formado por quatro linhas produtivas e em cada uma delas está instalada uma sequência de equipamentos que atendem a fabricação de todos os produtos, além de 21 funcionários com jornada de trabalho de 8 horas e 48 minutos de segunda à sexta-feira. O setor tem uma demanda média de 575 toneladas/mês, e na época do registro de dados esta demanda era atingida. Sendo necessário qualquer aumento acima dessa demanda média, horas extras precisam ser realizadas, gerando gastos operacionais adicionais e assim reduzindo as margens de lucro da empresa. A Figura 2 mostra a esquematização dos processos das linhas de manufatura.

Figura 02 - Esquema do processo produtivo na época da coleta de dados

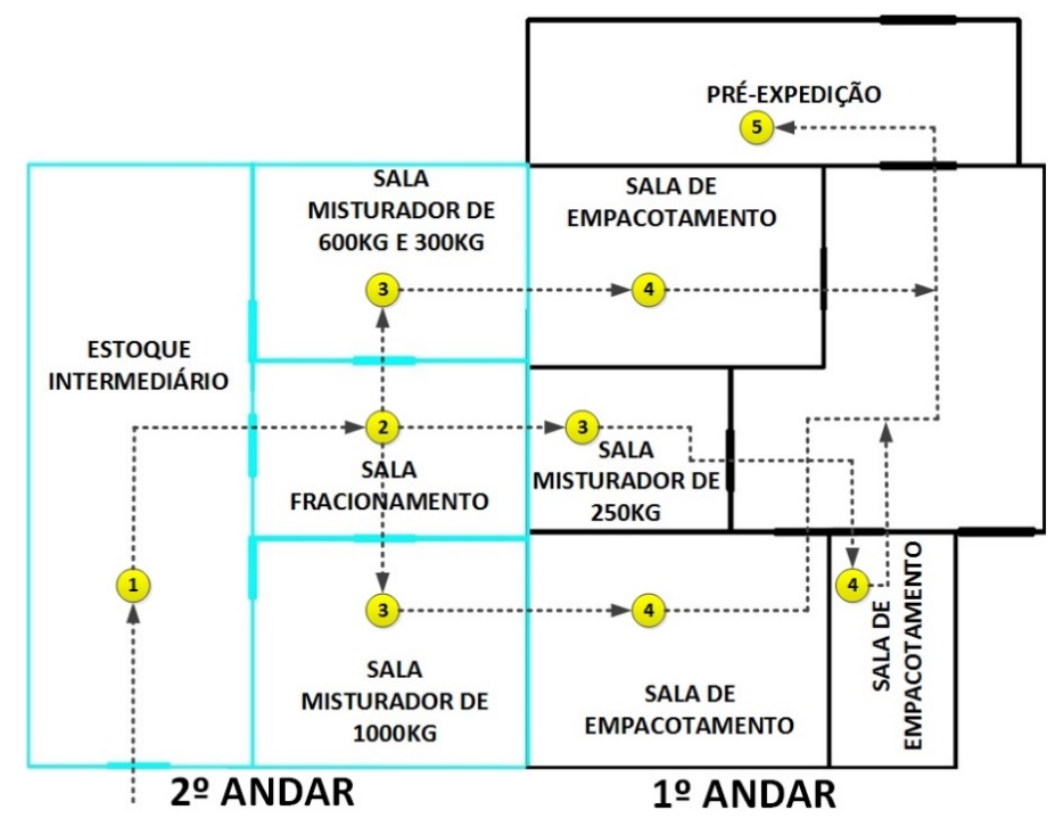

Fonte: Elaborado pelo autor (2017)

Conforme representado na Figura 2 em (1) armazena-se a matéria-prima no estoque intermediário localizando dentro da área fabril até sua utilização; em (2) são fracionados os ingredientes conforme a receita de cada produto; em (3) os 
ingredientes são adicionados aos misturadores e liberados para o silo localizado abaixo do misturador no $1^{\circ}$ andar; em (4) o produto misturado é retirado do interior do silo e envasado em sacos de 20 e 5 quilogramas e armazenados em pallets; e finalmente em (5) a empilhadeira retira os pallets de produto finalizados os quais são levados até a área de pré-expedição.

\subsection{Definição do mix de produção}

Para a definição do mix produtivo, foram considerados todos os produtos vendidos entre os períodos de julho de 2015 a junho de 2016, e realizada a média mensal dos quilogramas vendidos, sendo os produtos classificados conforme 0 método da curva ABC (Tabela 1 ).

Tabela 1 - Classificação ABC dos produtos produzidos no processo produtivo

\begin{tabular}{cccc}
\hline Grupo & Número de produtos & $\begin{array}{c}\text { Total por grupo } \\
\text { ABC } \mathbf{( k g )}\end{array}$ & $\begin{array}{c}\text { \% por grupo } \\
\text { ABC }\end{array}$ \\
\hline A & 3 & $110.493,95$ & $19,25 \%$ \\
B & 15 & $173.512,10$ & $30,22 \%$ \\
C & 598 & $574.091,00$ & $50,53 \%$ \\
\hline TOTAL & 616 Produtos & $\mathbf{8 5 8 . 0 9 7 , 0 5}$ & $\mathbf{1 0 0 , 0 0 \%}$ \\
\hline
\end{tabular}

Fonte: Elaborado pelo autor (2017)

A Tabela 1 mostra que $19,25 \%$ dos quilogramas vendidos correspondem a somente três produtos, formando a classe A. Outros quinze itens representam 30,22\% e integram a classe B. Já a classe C, que representa 50,53\% dos quilogramas vendidos, é formada por 598 produtos diferentes.

Para este estudo foram considerados somente os dados dos itens da classe A e B por sua maior representatividade, já que totalizam 18 produtos diferentes e correspondem a 49,47\% da média de quilogramas vendidos mensalmente pela empresa.

\subsection{Projeto de layout funcional}

No projeto de layout funcional foram definidos cinco departamentos, sendo eles: o estoque, o fracionamento, a mistura e o envase. Este último foi dividido em duas partes, sendo a primeira denominada "envase", contemplando as operações de 
retirada do produto do silo e conferência do peso do pacote; e a segunda parte foi denominada "selagem", a qual contempla a vedação da embalagem e a montagem do pallet de produto acabado. Em seguida realizaram-se as medições, em metros quadrados, do espaço físico de cada uma das áreas considerando o espaço reservado no layout atual. Na etapa seguinte foi classificada a relação de proximidade entre todos os cinco departamentos, por grau de importância. Para tanto foram utilizadas as letras $A, E, I, O, U$ e X, as quais representam os graus de importância 6, 5, 4, 3, 2 e 1 , respectivamente (Figura 3 ).

Figura 3 - Grau de relação de proximidade entre departamentos

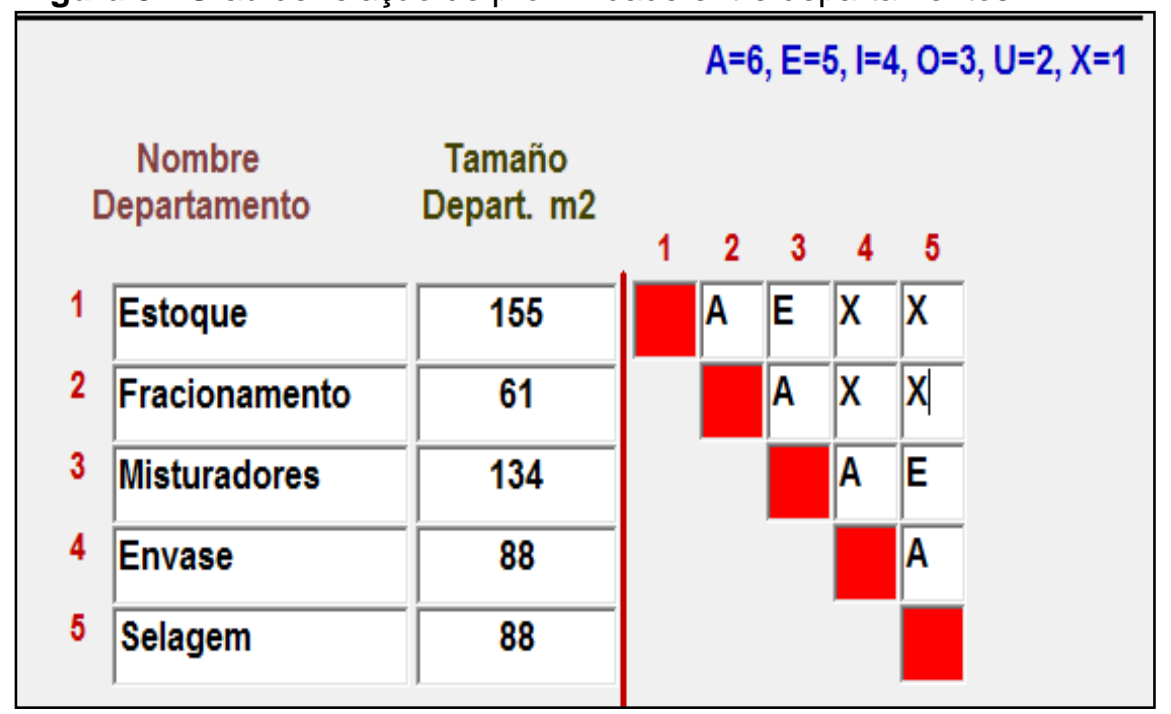

Fonte: Elaborado pelo autor (2017).

A classificação do grau de importância foi realizada junto com o gestor da área que apontou os principais pontos a serem considerados para o melhor fluxo do processo produtivo, assim como suas restrições. Desta forma é possível perceber que o estoque de matéria-prima tem grande importância de proximidade com 0 fracionamento e os misturadores, já que os dois últimos departamentos são abastecidos pelo estoque. Já o departamento de envase não tem qualquer necessidade de aproximação com o estoque e o fracionamento, sendo este departamento abastecido somente pelos misturadores. O mesmo acontece com a selagem, que é abastecida somente pelo envase. Considerando o layout ideal gerado pelo software CORELAP, foi projetado o desenho do arranjo físico do tipo funcional, conforme apresentado na Figura 4. 
Figura 4 - Layout funcional projetado (esquerda) em comparação com o layout atual (direita) LAYOUT FUNCIONAL
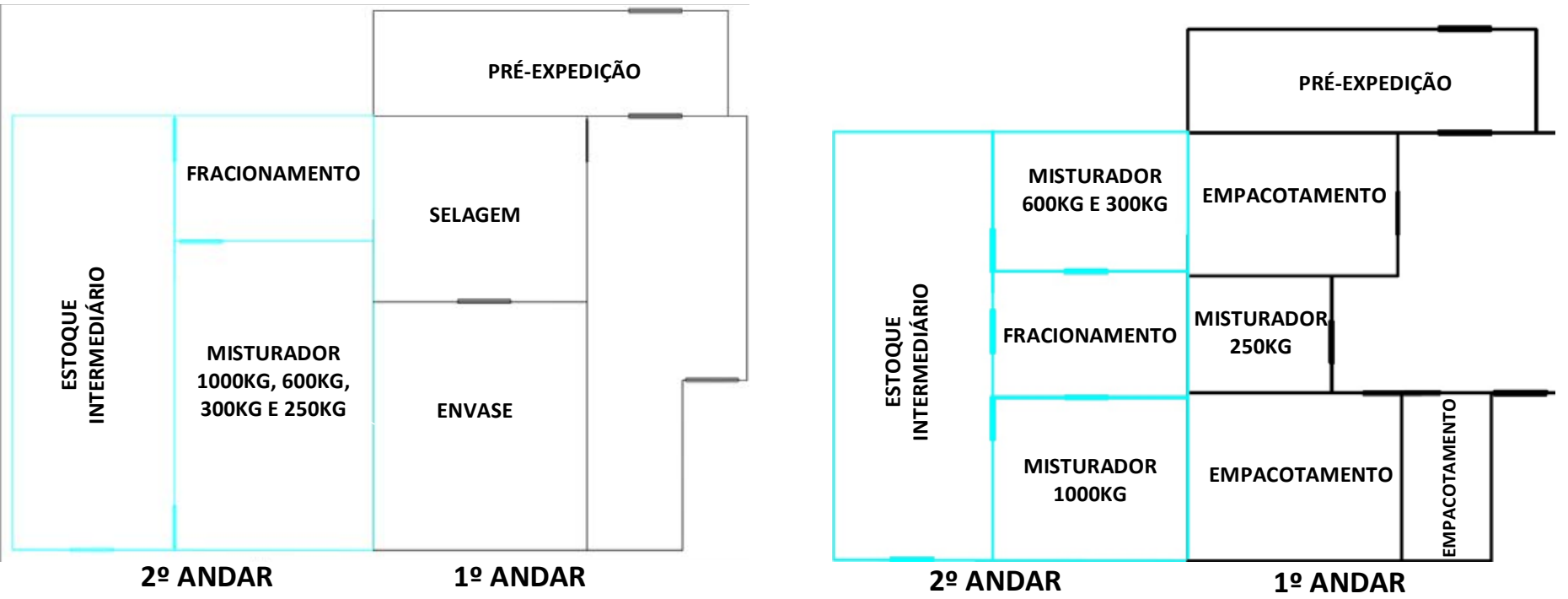

Fonte: Elaborado pelo autor (2017) 
No segundo andar foi mantido o estoque intermediário de matéria-prima no mesmo lugar, porém a sala de fracionamento foi retirada do centro e posicionada posteriormente aos misturadores. As duas salas destes misturadores foram unidas com o novo projeto, possibilitando que todos os equipamentos permaneçam juntos, até mesmo o misturador que ficava no primeiro andar.

No primeiro andar foi formada uma sala de envase que ficou posicionada abaixo dos misturadores, possibilitando às misturas, quando prontas, caírem nos silos. Ao lado da sala de envase ficou a sala de selagem, que possibilita a selagem dos produtos e a sua paletização. A pré-estocagem foi mantida no mesmo lugar.

\subsection{Projeto de layout celular}

Observou-se durante o estudo que no layout atual a distribuição física dos equipamentos e dos postos de trabalho já atendia à lógica de layout celular, apesar de não haver uma clara definição das famílias de produtos e onde elas deveriam ser produzidas. Assim, utilizando o método de análise de fluxo de produção (AFP), foram identificados os departamentos dentro do setor: estoque, fracionamento, mistura e envase. A seguir, foram elencados todos os equipamentos utilizados no processo, sendo estes relacionados com a necessidade na produção de cada item. Na segunda etapa, buscou-se o melhor agrupamento na formação de famílias para os produtos com base nos equipamentos utilizados em comum. A partir da análise de grupos, observou-se a formação de quatro células a partir dos grupos de equipamentos conforme similaridade. Os produtos a granel, como os sacos de 20 quilogramas, devem ser produzidos em duas das quatro células - célula 600 e 1000. Já os produtos fracionados, ou seja, em pacotes de cinco quilogramas, devem ser produzidos na célula 250 e 300, assim simplificando o fluxo de produção.

Considerando que a disposição atual dos equipamentos atende o layout celular, foi mantido para o projeto o mesmo posicionamento conforme apresentado na Figura 2, porém foi revista a necessidade de cada um dos equipamentos na linha produtiva. A tecnologia de grupos definiu as células dedicadas para cada um dos dois principais tipos de produtos: sacos a granel de 20 quilogramas e pacotes fracionados de cinco quilogramas. Desta forma não se propôs alteração no arranjo físico, porém 
houve alterações na padronização dos fluxos na produção de cada tipo de produto e na quantidade de equipamentos.

\subsection{Simulação dos modelos computacionais}

\subsubsection{Coleta e validação dos dados}

Os dados de entrada para a construção dos modelos foram levantados por meio de coleta de tempos no processo produtivo. Os tempos coletados foram analisados no software StatFit ${ }^{\circledR}$. Neste, foi analisado o grau de dispersão das amostras, através da construção de um diagrama Box Plot para cada grupo de amostras, a fim de eliminar os valores discrepantes (outliers). Após, o software calculou qual a distribuição probabilística mais adequada para cada uma das operações acompanhadas. É importante ressaltar que a análise da distribuição probabilística foi realizada considerando a distribuição contínua, já que os dados consistem em medidas de tempo.

A fim de validar os tempos de processamento, lógica de operações e lógicas de roteamento, buscou-se validar o modelo através da comparação dos resultados de produção média diária, dentro do período de três meses, de cada uma das quatro linhas produtivas em relação aos resultados de produção da simulação do sistema atual.

Observou-se que o gargalo produtivo com este mix de produtos é a operação de adição e mistura dos ingredientes. Seu tempo disponível, em um turno, é de 528 minutos, dividido pelo tempo de operação de cada ciclo, 18 minutos. Após, este resultado foi multiplicado pela capacidade de cada um dos misturadores de 250, 300, 600 e 1000 quilogramas. Por fim, este valor foi multiplicado pelo índice histórico de eficiência de cada uma das linhas, assim resultando na produção média/dia de cada uma delas.

Após definida a produção planejada para cada linha, estes resultados foram comparados ao sistema atual simulado. Desta forma foram verificadas divergências entre os dois sistemas (Tabela 2). 
Tabela 2 - Comparação entre a produção média diária e a produção diária simulada

\begin{tabular}{lllll}
\hline & \multicolumn{4}{c}{ Linha Produtiva } \\
& Linha 1000 & Linha 600 & Linha 300 & Linha 250 \\
\hline Produção Planejada $(\mathrm{kg})$ & $17.891,54$ & $10.912,00$ & $5.896,00$ & $4.913,33$ \\
Produção Simulada $(\mathrm{kg})$ & $17.920,00$ & $10.800,00$ & $5.725,00$ & $4.805,00$ \\
\hline Variação & $\mathbf{0 , 1 6 \%}$ & $\mathbf{- 1 , 0 3 \%}$ & $\mathbf{- 2 , 9 0 \%}$ & $\mathbf{- 2 , 2 0 \%}$ \\
\hline
\end{tabular}

Fonte: Elaborada pelo autor (2017)

A partir desta comparação considerou-se que as variações entre a produção planejada e a produção simulada ficaram baixas. Considerando estes índices, foi validado o sistema modelado.

\subsubsection{Construção e simulação do modelo computacional do layout Funcional}

A construção do modelo computacional do layout funcional foi realizada através do software ProModel ${ }^{\circledR}$. Conforme está representado na Figura 5, no departamento de fracionamento ficaram dois funcionários realizando a operação de fracionamento de ingredientes para todos os quatro misturadores, os quais agora estão agrupados juntos na mesma sala. Na sala de mistura, observando-se de baixo para cima, os misturadores tiveram a seguinte ordenação: misturador de 1000, 600, 300 e 250 litros. Foi mantido um operador exclusivo para os misturadores de 600 e 1000 litros, devido a seu maior tempo de adição dos ingredientes, que é realizado pelo operador. Já nos misturadores de 250 e 300 litros ficou um operador operando ambos, devido ao tempo inferior de adição de ingredientes, assim não repercutindo sobre a redução da produção. 
Figura 5 - Modelo computacional do layout funcional

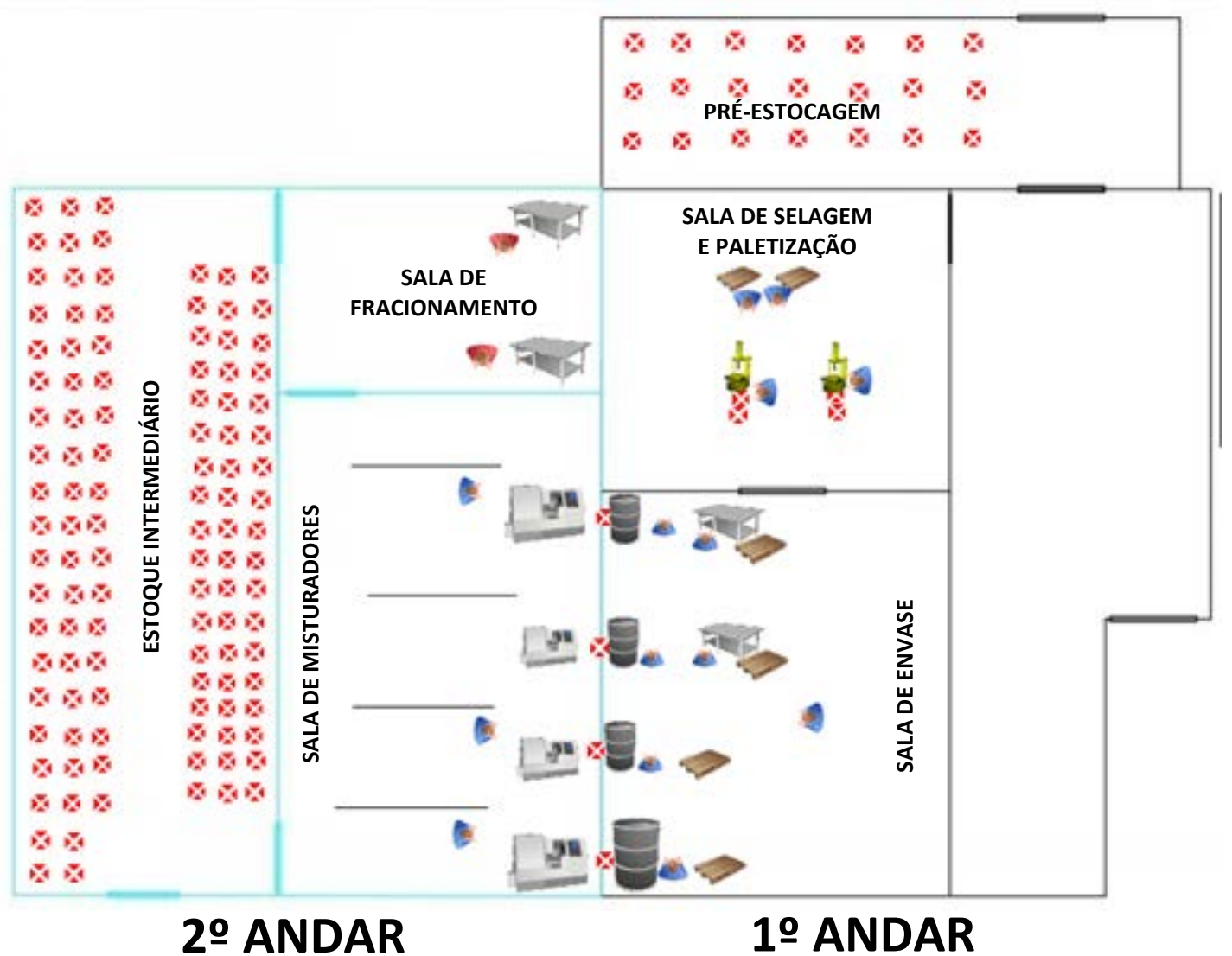

Fonte: Elaborado pelo autor (2017).

Silva e Rentes (2012) sugerem que produtos que compartilham uma mesma linha, porém com tempos de ciclo diferentes, sejam incluídos em famílias diferentes. Assim, no sistema simulado foi considerada a produção exclusiva dos produtos fracionados (pacotes de cinco quilogramas) nos misturadores de 250 e 300 litros e dos produtos a granel (sacos de 20 quilogramas) nos misturadores de 600 e 1000 litros. Esta definição foi considerada importante para o melhor sincronismo de produção, possibilitando a redução de setup, no número de equipamentos e na baixa variabilidade nos tempos de ciclo.

Nos silos localizados abaixo dos misturadores, foi realizado um sistema de roteamento das misturas, permitindo com que a mistura concluída possa ser enviada para qualquer um dos dois silos. Por exemplo, a mistura concluída no misturador de 1000 litros pode ser enviada tanto para o silo localizado abaixo do misturador de 1000 litros, como para o silo localizado abaixo do misturador de 600 litros, permitindo assim maior sincronia na produção, e aumentando a ocupação do departamento de envase. O mesmo acontece com os misturadores de 250 e 300 litros. 
No departamento de envase, foram montados - em frente de cada silo - centros de envase conforme a característica de empacotamento de cada produto, a granel ou fracionado. Em frente a cada um dos silos do misturador de 250 e 300 litros são necessários dois funcionários, um para a retirada do produto do silo e outro para a pesagem e colocação do produto em um pallet de armazenamento. Em frente a cada um dos silos dos misturadores de 600 e 1000 litros é necessário um funcionário, o qual retira o produto do silo, acerta o peso na embalagem e coloca o produto em um pallet de armazenamento. Para a movimentação destes pallets de armazenamento até o próximo departamento foi considerado um funcionário que realiza

exclusivamente o transporte dos pallets de produtos armazenados para 0 departamento de selagem.

No departamento de selagem, foram considerados dois centros, cada um com duas seladoras contínuas, duas seladoras intermitentes e um funcionário para realizar as operações de selagem. São necessários dois locais de paletização, um para a paletização de produtos a granel e outro para produtos fracionados, assim mantendo um funcionário para cada local para realizar suas operações.

O funcionário que realiza o transporte deixa o pallet de produtos em frente ao centro de selagem que estiver disponível primeiro, e retorna ao seu departamento. Os centros de selagem realizam suas operações que por fim entregam o produto selado aos respectivos locais de paletização. Após montados os pallets, os mesmos são retirados pela empilhadeira e levados até a pré-estocagem.

Para a validação do quadro de funcionários para esta modelagem, o sistema simulado e os resultados obtidos das análises dos recursos fornecidas pelo software ProModel ${ }^{\circledR}$ foram apresentados para avaliação aos gestores do setor.

\subsubsection{Construção e simulação do modelo computacional do layout celular}

A construção do modelo computacional do layout celular foi realizada considerando a mesma distribuição dos equipamentos do sistema atual do setor, porém houve mudanças no fluxo dos produtos produzidos e na quantidade de equipamentos em cada célula (Figura 5 ). 
Figura 5 - Modelo computacional do layout celular

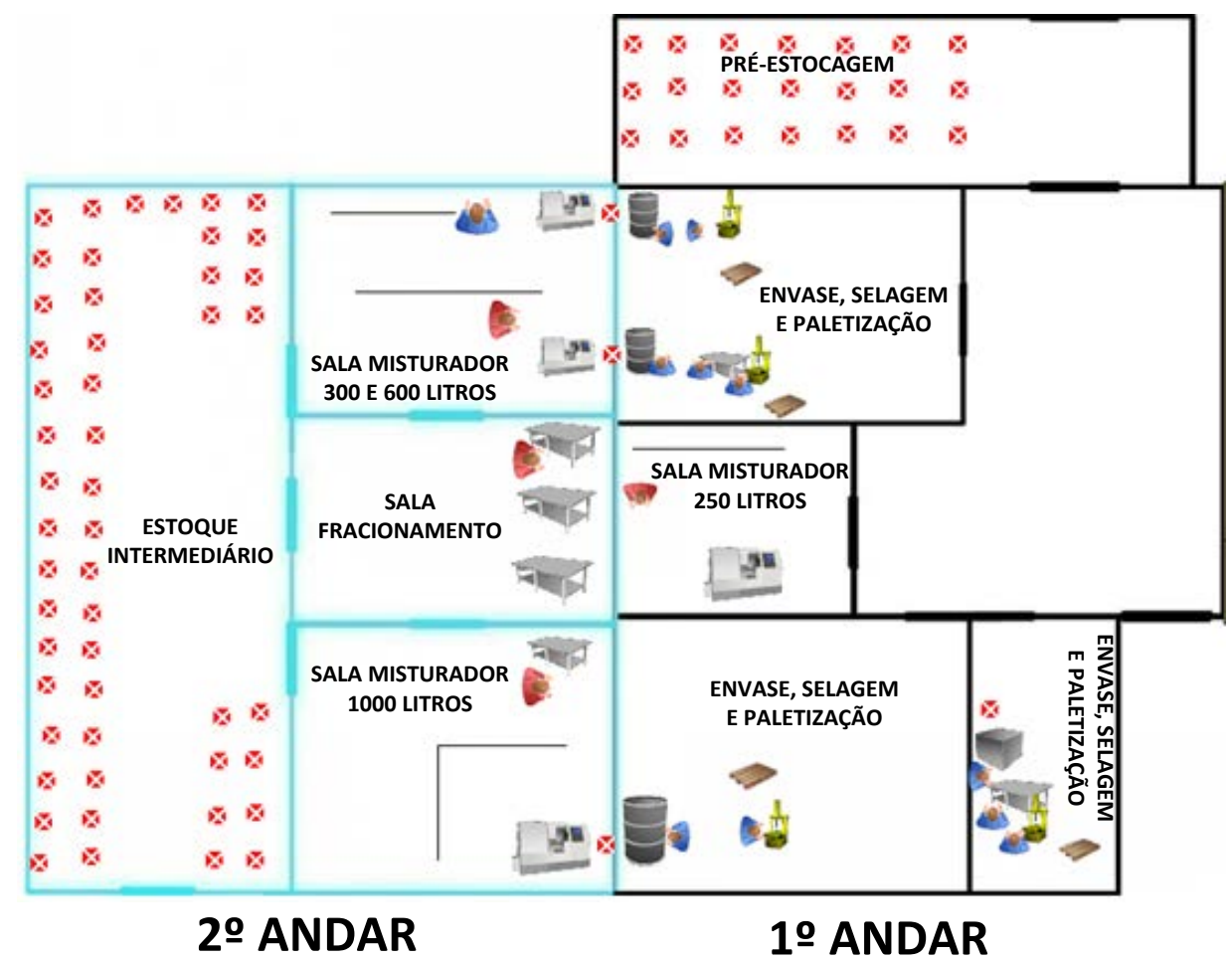

Fonte: Elaborado pelo autor (2017)

As células dos misturadores de 250 e 300 litros foram destinadas para a produção exclusiva de produtos fracionados (pacotes de 5 quilogramas) e as células dos misturadores de 600 e 1000 litros, para a produção de produtos a granel (sacos de 20 quilogramas).

Quanto ao quadro de funcionários, foi necessário realizar diversos rearranjos. No fracionamento foram mantidos os três centros de trabalho, porém foi conservado apenas um funcionário fixo realizando a operação de fracionamento exclusivamente para o misturador de 600 litros, porque o operador desta célula encontrava-se sobrecarregado, tornando-se gargalo do sistema produtivo. Para o fracionamento dos ingredientes dos demais misturadores seus respectivos operadores devem realizar tal função.

Tubino (1999) salienta que há diversas formas de células de manufatura as quais podem otimizar o fluxo de pessoas e componentes, assim como reduzir o tempo de movimentação. Seguindo esta lógica, no primeiro andar, foi reduzido um funcionário de cada célula de envase. O funcionário que realiza a operação de selagem do produto também executa a paletização do produto, devido à aproximação entre os dois centros produtivos, a seladora e o palete. 
Para a validação do quadro de funcionários para esta modelagem, o sistema simulado foi apresentado aos gestores do setor e foram avaliados os resultados obtidos das análises dos recursos fornecidas pelo software ProModel®.

\subsection{Comparação entre os layouts sob a ótica dos indicadores da TOC}

As sessões a seguir abordam a comparação entre os layouts sob a ótica dos indicadores da TOC.

\subsubsection{Cálculo do indicador de Investimentos (I)}

O indicador de investimentos é formado por dois grupos: o de matérias-primas e de investimentos. Para isso foram listados todos os equipamentos do setor e as matérias-primas em estoque, considerando o seu valor monetário.

Com as simulações foi possível determinar a quantidade de equipamentos necessária para cada um dos modelos simulados, de forma que se pôde definir o valor de investimentos para cada um dos três modelos e fazer um comparativo de entre eles.

\subsubsection{Cálculo do indicador de Despesas Operacionais (DO)}

Para o cálculo do indicador de despesas operacionais, considerando que todos os equipamentos do setor produtivo já foram elencados, foi verificado junto ao setor de contabilidade o valor de depreciação mensal dos mesmos. Considerando a quantidade de equipamentos para cada um dos três layouts, foi possível calcular o valor de depreciação para cada um deles.

Além da soma da depreciação, para o cálculo do indicador da DO também foi necessário quantificar a soma das despesas operacionais mensais de cada layout. Estas despesas são formadas por quatro grupos: pessoal direto, gastos indiretos, materiais indiretos e gastos indiretos. 


\subsubsection{Cálculo do indicador Ganho (G)}

Para o cálculo do indicador operacional de Ganho, foram levantados os preços de venda dos produtos do mix definido, e seus custos totalmente variáveis, ou seja, os custos de matéria-prima, para o cálculo do ganho unitário de cada produto. Após, foi necessário determinar a quantidade produzida de cada produto. Para isso, foram utilizados os dados da produção média para o layout atual. Já para os layouts funcional e celular, foram utilizados os resultados obtidos com a simulação de produção.

Após definidos a quantidade para cada produto e o ganho unitário de cada um deles, o ganho total dos produtos foi calculado bem como o ganho total de cada layout, multiplicando a quantidade produzida em quilogramas/dia pelo ganho unitário do produto.

Ao final tem-se o resultado dos três indicadores operacionais da TOC, na sequência do seu grau de prioridade sob a ótica da TOC, referente a cada um dos três layouts, além da lógica do indicador, de forma que quando há o sinal de subtração (-) a lógica do indicador é quanto menor melhor e quando há o sinal de soma (+), a lógica é quanto maior melhor (Tabela 3).

Tabela 3 - Resultados dos indicadores operacionais da TOC para os três layouts

\begin{tabular}{|c|c|c|c|c|c|c|c|}
\hline $\begin{array}{l}\text { Lógica do } \\
\text { Indicador }\end{array}$ & Indicador & \multicolumn{2}{|r|}{$\begin{array}{l}\text { Layout } \\
\text { Atual }\end{array}$} & \multicolumn{2}{|c|}{$\begin{array}{l}\text { Layout } \\
\text { Funcional }\end{array}$} & \multicolumn{2}{|c|}{$\begin{array}{l}\text { Layout } \\
\text { Celular }\end{array}$} \\
\hline+ & Ganho (G) & $\mathrm{R} \$$ & 26.504 & $\mathrm{R} \$$ & 29.775 & $\mathrm{R} \$$ & 26.540 \\
\hline- & Investimento (I) & $\mathrm{R} \$$ & 767.910 & $\mathrm{R} \$$ & 679.834 & $\mathrm{R} \$$ & 707.886 \\
\hline- & Despesa Operacional (DO) & $\mathrm{R} \$$ & 162.617 & $\mathrm{R} \$$ & 132.181 & $\mathrm{R} \$$ & 126.973 \\
\hline
\end{tabular}

Fonte: Elaborado pelo autor (2017).

\section{CONCLUSÃo}

Ao avaliar o resultado dos três indicadores e respeitando o grau de prioridade de cada um deles, constatou-se que o layout funcional apresentou o melhor desempenho, pois obteve melhores resultados nos dois indicadores de maior prioridade, ganho e investimento. 
Constatou-se que o resultado do indicador de ganho foi superior no layout funcional devido ao aumento da flexibilidade do fluxo entre os departamentos produtivos, de forma que os produtos manufaturados obtiveram a liberdade de seguir para etapa seguinte, a qual equipamento ou recurso estivesse liberado primeiro, sem ficar atrelado a um fluxo único no processo. Isto possibilitou a maior utilização dos equipamentos e dos recursos e, em decorrência, reduziu sua ociosidade e tempos de espera.

A flexibilidade entre os departamentos gerada pelo layout funcional também impactou na utilização mais balanceada dos equipamentos. Desta forma o indicador de investimentos do layout funcional apresentou um desempenho superior em relação aos demais.

Tanto no layout funcional como no celular foi obtida uma redução considerável dos recursos (número de funcionários necessários) o que impactou diretamente no indicador de despesas operacionais. Isto foi alcançado através da reorganização do fluxo dos produtos a granel e fracionados através das operações necessárias para sua manufatura, destinando a produção exclusiva a determinados equipamentos, assim permitindo maior balanceamento dos recursos conforme a necessidade de cada produto.

Assim, percebe-se que a simulação é uma ferramenta versátil com a possibilidade de aplicações variadas no campo produtivo. Sua aplicação permitiu a avaliação dos impactos gerados pelas alterações propostas, tais como: rearranjo do posicionamento físico e redimensionamento da quantidade dos equipamentos e do quadro funcional sem a necessidade de grandes investimentos.

Os modelos simulados possibilitaram avaliar o nível de produção que pode ser atingido com os diferentes layouts, diante dos recursos disponibilizados e das restrições consideradas. Também permitiram a otimização das variáveis, reduzindo os erros de avaliação cometidos ao se analisar taxas médias de eventos em vez de distribuições probabilísticas. Além disso, facilitaram a visualização do funcionamento dos sistemas, viabilizando uma melhor percepção, para gestores da empresa, de como os modelos propostos funcionariam em uma situação real, facilitando a aprovação das mudanças, sem a necessidade de interferência na linha produtiva atual. 
Por fim, o trabalho procurou contribuir para construção de um elo entre a abordagem do projeto de layout e os indicadores da TOC por meio da utilização da simulação de eventos discretos. Assim os indicadores operacionais da TOC se mostraram eficazes em determinar o layout que ofereceria maior vantagem competitiva à empresa. Os indicadores também tiveram adequada aplicabilidade no sistema produtivo, demonstrando a versatilidade para possivelmente quaisquer outros sistemas, no apoio a decisão de forma empírica, sem a perda no foco da meta da empresa, o lucro. Sua aplicação também permitiu garantir o ótimo global, e não apenas o ótimo local, que segundo a TOC, é o que deve ser buscado, verificando que qualquer despesa operacional somente seja feita caso não comprometa o ganho, ou caso a despesa operacional seja superior ao ganho reduzido pela mudança.

Por fim, através deste estudo foi possível demonstrar que a interlocução entre a simulação de eventos discretos e os indicadores operacionais da Teoria das Restrições (TOC) é uma interessante e inovadora abordagem no projeto e análise de layout, não sendo encontradas publicações semelhantes na literatura atual.

\section{REFERÊNCIAS}

ALMEIDA, M. S. et. al. Utilização da simulação em ARENA 7.0 no auxílio ao balanceamento da célula de montagem de uma fábrica de calçados. XXVI Encontro Nacional de Engenharia de Produção - ENEGEP, 2006.

ANTUNES, J. et. al. Sistemas de produção: conceitos e práticas para projetos e gestão da produção enxuta. Porto Alegre: Bookman, 2008.

ARAÚJO, R. Modelo de simulação para avaliação do fluxo de transporte rodoviário de sondas de perfuração terrestre de poços de petróleo. 2002. 144 f. Dissertação (Mestrado em Engenharia da Produção) - Universidade Federal de Santa Catarina, Florianópolis, 2002.

ASKIN, R; GOLDBERG, J. Design and analysis of lean production systems. New York: John Wiley \& Sons Inc, 2002.

BATEMAN, R. et. al. Sistemas de simulação: aprimorando processos de logística, serviços e manufatura. Rio de Janeiro: Elsevier, 2013.

BANKS, J. Handbook of simulation: Principles, Methodology, Advances, Applications and Practice. New York: John Wiley \& Sons Inc, 1998.

https://doi.org/10.1002/9780470172445 
BLACK, J. O Projeto da fábrica com futuro. Porto Alegre: Bookman, 1998.

BURBIDGE, J. The Introduction of group technology. London: Heinemann, 1975.

BURBIDGE, J. Planejamento e controle da produção. São Paulo: Atlas, 1981.

BOBLITZ, G. Simulation eliminates need for na $\$ 80,000$ machine and conveyor investment. Industrial Engineering, v. 23, n. 3, p. 26-28, 1991.

CHASE, R.; JACOBS, R.; AQUILANO, N. Administração da Produção Para a Vantagem Competitiva. 10. ed. Porto Alegre: Bookman, 2006.

CHWIF, L.; MEDINA, A. Modelagem e simulação de eventos discretos: teoria e aplicações. 3. ed. São Paulo: Edição do Autor, 2010.

CORRÊA, H.; CORRÊA, C. Administração de produção e de operações:

manufatura e serviços: uma abordagem estratégica. 2. ed. São Paulo: Atlas, 2006.

DE LIMA, D; et al. Mapeamento do fluxo de valor e simulação para implementação de práticas lean em uma empresa calçadista. Revista Produção Online, Florianópolis, v. 16, n. 1, p. 366-392, mar. 2016.

https://doi.org/10.14488/1676-1901.v16i1.2183

DIAS, S.; et al. Alinhamento entre sistemas de produção, custo e indicadores de desempenho: um estudo de caso. Revista Produção Online, Florianópolis, v. 7, n. 2, p 144-169, ago. 2007.

https://doi.org/10.14488/1676-1901.v7i2.72

DINIS-CARVALHO, J.; ALVES, A.; SOUSA, R. Moving from job-shop to production cells without losing flexibility: a case study from the wooden frames industry. South African Journal of Industrial Engineering, Pretoria, v. 25, n. 3, p 212-225, nov. 2014. https://doi.org/10.7166/25-3-864

FILHO, P. Introdução à modelagem e simulação de sistemas: com aplicações em arena. Florianópolis: Visual Books Ltda., 2001.

GAITHER, N.; FRAZIER, G. Administração da produção e operações. 8. ed. São Paulo: Pioneira, 2002.

GROOVER, M. Automation, production system and computer-integrated manufacturing. 2. ed. Upper Saddle River: Prentice Hall, 2001.

GOLDRATT, E. A síndrome do palheiro: garimpando informações num oceano de dados. São Paulo: Educator, 1996.

GOLDRATT, E.; COX, J. A meta: um processo de melhoria contínua. 2. ed. São Paulo: Nobel, 2002.

GOLDRATT, E.; FOX, R. A corrida pela vantagem competitiva. São Paulo: Educator, 1989. 
KRAJEWSKI, L.; RITZMAN, L.; MALHOTRA, M. Administração de produção e operações. 8. ed. São Paulo: Pearson Prentice Hall, 2009.

LAW, A. M.; KELTON, W. D. Simulation Modeling \& Analysis. 2. ed. New York: McGraw-Hill Books, 1991.

LI, Jing-Wen. Investigating the efficacy of exercising JIT practices to support pull production control in a job shop environment. Journal of Manufacturing

Technology Management, v. 16, n. 7, p. 765-783, 2005.

https://doi.org/10.1108/17410380510626187

LOPES, A. Avaliação de capacidade de fluxo: sistemas de armazenagem automático. Anais da $\mathbf{1}^{\mathrm{a}}$ Conferência Latino Americana de Simulação e Usuários Promodel, 1999.

MEIRELLES, A. et al. Simulação e Layout: Um Estudo de Caso. ENCONTRO NACIONAL DE ENGENHARIA DE PRODUÇÃO-ENEGEP, 29., 2009. Anais...2009.

MOREIRA, D. Administração da produção e operações. 2. ed. São Paulo: Cengage Learning, 2008.

NEUMANN, C.; SCALICE, R. Projeto de fábricas e layout. Rio de Janeiro: Elsevier, 2015.

PEINADO, J.; GRAEML, A. Administração da produção: operações industriais e de serviços. Curitiba: UnicenP, 2007.

PEREIRA, C.; COSTA, M. Um modelo de simulação de sistemas aplicado à programação da produção de um frigorífico de peixe. Revista Produção Online, Florianópolis, v. 12, n. 4, p. 972-1001, dez. 2012.

https://doi.org/10.14488/1676-1901.v12i4.994

PINHO, A., et. al. Aumento de Produtividade em uma Linha de Montagem de Chassis Automotivos Através da Simulação Computacional. ENCONTRO NACIONAL DE ENGENHARIA DE PRODUÇÃO, v. 26, 2006.

RITZMAN, L.; KRAJEWSKI, L. Administração da Produção e Operações. São Paulo: Pearson Prentice Hall, 2004.

SANTOS, L.; GOHR, C.; URIO, L. (2014). Planejamento sistemático de layout em pequenas empresas: uma aplicação em uma fábrica de baterias automotivas.

Espacios, v. 35, n. 7, 2014.

Silva, J., et. al. Planejamento Sistemático do Layout: aplicação em uma indústria de panelas esmaltadas. Espacios, v. 36, n. 9, 2015.

SILVA, A.; RENTES, A. Um Modelo de Projeto de Layout Para Ambientes Job Shop com Alta Variedade de Peças Baseado nos Conceitos da Produção Enxuta. Gestão 
\& Produção, v. 19, n. 3, p. 531-541, 2012.http://dx.doi.org/10.1590/S0104$\underline{530 \times 2012000300007}$

SLACK, N., et. al. Administração da produção. 3. ed. São Paulo: Atlas, 2009.

SOARES, J., et. al. A contribuição da simulação computacional para a análise sistêmica da reestruturação de layout e otimização de recursos na manufatura celular: estudo de caso em uma célula de uma empresa do ramo automotivo.

Produto \& Produção, v. 12, n. 3, 49-68, 2011.

https://doi.org/10.22456/1983-8026.22802

TOLLINGTON, T. ABC vs. TOC: Same Cloth as Absorption vs. Marginal, Different Style and Cut. Management Accounting, v. 76, n. 4, p 44, 1998.

TUBINO, D. Sistemas de Produção: a produtividade no chão-de-fábrica. Porto Alegre: Bookman, 1999.

WEMMERLÖV, U.; JOHNSON, D. Cellular Manufacturing at 46 User Plants: Implementation Experiences And Performance Improvements. International Journal of Production Research, v. 27, n. 9, p. 1511-1530, 1997.

https://doi.org/10.1080/002075497195966

XAVIER, A., et. al. A simulação a eventos discretos como ferramenta de tomada de decisão na implementação de uma linha de montagem em uma indústria do setor automobilístico. Revista Inovação, Gestão e Produção, v. 2, n. 5, p. 1-15, 2010.

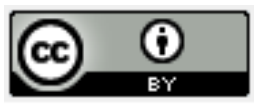

Artigo recebido em: 20/01/2018 e aceito para publicação em: 12/02/2019

DOI: http://dx.doi.org/10.14488/1676-1901.v19i1.3147 